\title{
NCLA Executive Board Directory, 2006-2007
}

\section{OFFICERS}

President

Dr. Robert Burgin

307 Swiss Lake Dr.

Cary, NC 27513

rburgin@mindspring.com

Phone: (919) 462-0134

Vice President/ President Elect

Phil Barton

Rowan Public Library

P.O. Box 4039

Salisbury, NC 28145

bartonp@co.rowan.nc.us

Phone: (704) 216-8229

Secretary

Paula Hinton

P.O. Box 1148

Buies Creek, NC 27506

pphinton@email.unc.edu

Phone: (919) 962-1151

Treasurer

Tina Stepp

45 Kingswood Drive

Hendersonville, NC 28792

tstepp@henderson.lib.nc.us

Phone: (828) 697-4725

Directors

Frannie Ashburn

State Library of North Carolina

4640 Mail Service Center

Raleigh, NC 27699

FAshburn@library.dcr.state.nc.us

Phone: (919) 807-7416

Bryna Coonin

Joyner Library

North Carolina Collection

East Carolina University

Greenville, NC 27858

COONINB@MAIL.ECU.EDU

Phone: (252) 328-0431

ALA Council

Kevin Cherry

124 E. 2nd St.

Washington, NC 27889

CHERRYT@MAIL.ECU.EDU

SELA Rep

Evelyn Council

Chesnutt Library

Fayetteville State University

3429 Sandystone Circle

Fayetteville, NC 28311

ecouncil@uncfsu.edu

Phone: (910) 672-1539
Editor, NCL

Ralph Scott

Academic Library Services

4016 Joyner Library

East Carolina University

Greenville, NC 27858

scottr@mail.ecu.edu

Phone: (252) 328-0265

Fax: (252) 328-0268

Past President

Dr. Pauletta Brown Bracy

North Carolina Central University

School of Library and Information

Sciences

PO Box 19586

Durham, NC 27707

pbracy@nccu.edu

Phone: (919) 530-6401

Fax: (919) 530-6402

Administrative Assistant

(ex officio)

Kim Parrott

North Carolina Library Association

1811 Capital Blvd.

Raleigh, NC 27604

nclaonline@ibiblio.org

Phone: (919) 839-6252

Fax: (919) 839-6253

\section{SECTION CHAIRS}

BLINC

Susan Wolf Neilson

Cameron Village Regional Library

1930 Clark Avenue

Raleigh, NC 27605

susan.neilson@co.wake.nc.us

Phone: (919) 856-6718

CSS

Loree Kelly

EAST Regional Branch

4809 Clinton Road

Fayetteville, NC 28301

loreepkelly@netscape.com

Phone: (910) 485-2955

CUS

Tommy Nixon

Davis Library

University of North Carolina at Chapel Hill

CB\# 3922

Chapel Hill, NC 27514

tommy_nixon@unc.edu

Phone: (919) 962-1151

CJCLS

Debbie Luck

Randolph Community College Library

P. O. Box 1009

Asheboro, NC 27204

dsluck@randolph.edu

(336) 633-0204
GRS

2006: Joyce (Beth) Kaylor

Randall Library

University of North Carolina at

Wilmington

601 S. College Road

Wilmington, NC 28403

kaylorj@uncw.edu

Phone: (910) 962-4232

GRS

2007: Mimi Curlee

Public Library of Charlotte \&

Mecklenburg County

310 N. Tryon Street

Charlotte, NC 28202

MCurlee@plcmc.org

Phone: (704) 336-2725

LAMS

Mary Sizemore

Appalachian Regional Library

215 10th Street

North Wilkesboro, NC 28659

msizemore@arlibrary.org

Phone: (336) 838-2818

NCASL

Catherine Edwards-Spratley

Cardinal Gibbons High School

1401 Edwards Mill Road

Raleigh, NC 27607

cates_cghs@yahoo.com

Phone: (919) 834-1625 ext. 272

NCPLTA

Mary Hatcher

1905 E. Lake Shore Drive

Wilmington, NC 28401

M03477@aol.com

Phone: (910) 762-7259

PLS

Lynn Thompson

Southern Pines Public Library

170 W Connecticut Ave

Southern Pines, NC 28387

thompson@suppl.net

Phone: (910) 692-8235

RASS

Paula Hinton

P.O. Box 1148

Buies Creek, NC 27506

pphinton@email.unc.edu

Phone: (919) 962-1151

RTSS

Elizabeth Cramer

Appalachian State University Library

Boone, NC 28607

crameree@appstate.edu

Phone: (828) 262-4967 


\section{ROUND TABLE CHAIRS}

Literacy

Betty Meehan-Black

2302 Wade Hampton Road

Hillsborough, NC 27278

bmblack@email.unc.edu

Phone: (919) 962-1120

\section{NMRT}

Kaye Cook

Lewisville Branch Library

Lewisville Plaza Shopping Center

Lewisville, NC 27023

kayecook11@aol.com

Phone: (336) 945-3786

NCLPA

Jackie Cornette

Watauga County Library

1085 Old US 421

Sugar Grove, NC 28679

jcornette@arlibrary.org

Phone: (828) 297-5515

REMCO

H. Jamane Yeager

Belk Library

P.O. Box 2725

Reidsville, NC 27320

jyeager@elon.edu

Phone: (336) 278-6576

RTSC

Deborah Rouse

Rowan Public Library

201 W. Fisher St, P.O. Box 4039

Salisbury, NC 28145

RouseDH@co.rowan.nc.us

Phone: (704) 638-3020

RTSWL

Kathy Crowe

Jackson Library

University of North Carolina at Greensboro

Box 26170

Greensboro, NC 27402

kmcrowe@uncg.edu

Phone: (336) 256-0274

TnT

Beth Bernhardt

Jackson Library

University of North Carolina at Greensboro

Box 26170

Greensboro, NC 27402

Beth_Bernhardt@uncg.edu

Phone: (336) 256-1210

\section{COMMITTEE CHAIRS}

Archives

Jean Rick

Meredith College

Carlyle Library

3800 Hillsborough St.

Raleigh NC 27607

rickj@meredith.edu

Phone: (919) 760-8383
Conference 2007

Phil Barton

Rowan Public Library

P.O. Box 4039

Salisbury, NC 28145

bartonp@co.rowan.nc.us

Phone: (704) 638-3004

Constitution, Codes and Handbook

Bobby Wynn

Charles W. Chestnutt Library

Fayetteville State University

1200 Murchison Road

Fayetteville, NC 28301

bwynn@uncfsu.edu

Phone: (910) 672-1232

Continuing Education

Sherwin Rice

Bladen Community College

P.O. Box 266

Dublin, NC 28332

srice@bladen.cc.nc.us

Phone: (910) 879-5641

Endowment

David Goble

3217 Selwyn Farms Lane

Charlotte, NC 28209

David.Goble@cpcc.edu

Phone: (704) 330-6441

Fax: (704) 330-6887

Finance

Wanda Brown

Z. Smith Reynolds Library

Wake Forest University

Box 7777 Reynolda Station

Winston-Salem, NC 27109

brownw@wfu.edu

Phone: (336) 758-5094

Intellectual Freedom

jim Kuhlman

UNC-Asheville

Box 1500

Asheville, NC 28804

kuhlman@unca.edu

Phone: (828) 251-6545

Leadership Institute

Barbara Beebe

Davis Memorial Library

5400 Ramsey Street

Fayetteville, NC 28311

beebe@methodist.ecu

Phone: (910) 630-7618

Membership

Caroline Walters

1110 W Murray Ave.

Durham, NC 27704

carolinejwalters@netzero.com

Phone: [home] (919) 220-3197, [work] 919-807-7360
Nominating

Dr. Pauletta Brown Bracy

North Carolina Central University

School of Library and Information Sciences

PO Box 19586

Durham, NC 27707

pbracy@nccu.edu

Phone: (919) 530-6401

Fax: (919) 530-6402

Operations

Dale Cousins

Wake County Public Library

Cameron Village Regional Library

1930 Clark Ave.

Raleigh, NC 27605

gcousins@co.wake.nc.us

Phone: (919) 856-6726

Fax: (919) 856-6722

Public Policy

Ross Holt

Randolph County Public Library

201 Worth Street

Asheboro, NC 27203

rholt@randolphlibrary.org

Phone: (336) 318-6806

Fax: (336) 318-6823

Publications and Marketing

Suzanne White

Rowan Public Library

P.O. Box 4039

Salisbury, NC 28145

whites@co.rowan.nc.us

Phone: (704) 216-8242

Fax: (704) 216-8262

Scholarships

Harry Cooke

Gaston College

2425 31st St Dr NE

Hickory, NC 28601

cooke.harry@gaston.cc.nc.us

Phone: (704) 922-6355

Web Site

Bao-Chu Chang

NCSU Libraries

Campus Box 7111

Raleigh, NC 27695-7111

bao-chu_chang@ncsu.edu

Phone: (919) 515-2669

Fax: (919) 515-7292 


\section{EDITOR}

Ralph Lee Scott

Joyner Library

East Carolina University

Greenville, NC 27858

Tel: (252) 328-0265

Fax: (252) 328-6222

E-mail: scottr@mail.ecu.edu

\section{ASSOCIATE EDITOR}

Elizabeth H. Smith

Professor Emeritus

Joyner Library

East Carolina University

Greenville, NC 27858

Tel:(252) 328-0297

Fax:(252) 329-0868

E-mail: smithe@mail.ecu.edu

\section{ASSOCIATE EDITOR AND INDEXER}

Mike Van Fossen

Reference-Documents

Davis Library CB\# 3912

UNC-Chapel Hill

Chapel Hill, NC 27599-8890

Tel: (919) 962-1151

Fax: (919) 962-5537

E-mail: mike_vanfossen@unc.edu

\section{BOOK REVIEWS EDITOR}

Nicholas Graham

Head of Public Services

North Carolina Collection

UNC-Chapel Hill, NC 27514-8890

Tel: (919) 962-1172

Fax: (919) 962-4452

E-mail: ngraham@email.unc.edu

\section{ASSOCIATE EDITOR FOR RASS}

Joline R. Ezzell

Reference

Box 90175

Duke University Library

Durhan, NC 27708-0175

Tel: (919) 660-5925

Fax: (919) 684-2855

E-mail: joline.ezzell@duke.edu

\section{LAGNIAPPE EDITOR}

Joseph Thomas

Reference Librarian

Joyner Library

East Carolina University

Greenville, NC 27858

Tel: (252) 328-2266

Fax: (252) 328-2271

Email: thomasw@mail.ecu.edu

WIRED TO THE WORLD EDITOR

Ralph Lee Scott

Joyner Library

East Carolina University

Greenville, NC 27858

Tel: (252) 328-0265

Fax: (252) 328-6222

E-mail: scottr@mail.ecu.edu

\section{ASSOCIATE EDS. FOR ACADEMIC} LIBRARIES

Paula P. Hinton

Reference Dept., CB\# 3922

Davis Library

UNC-Chapel Hill

Chapel Hill, NC 27514-8890

Tel: (919) 962-1151

Fax: (919) 962-5537

E-mail: pphinton@email.unc.edu

Page Life

Catalog Dept., CB\# 3914

Davis Library

UNC-Chapel Hill

Chapel Hill, NC 27514-8890

Tel: (919) 962-0153

Fax: (919) 962-4450

E-mail: pagelife@email.unc.edu
ASSOCIATE ED. FOR PUBLIC LIBRARIES

Suzanne White

Rowan Public Library

PO Box 4039

Salisbury, NC 28145

Phone: (704) 638-3009

ASSOCIATE ED. FOR SCHOOL LIBRARIES

Diane Kester

Associate Professor Emeritus

East Carolina University

105 Longview Drive

Goldsboro, NC 27534

Tel: (919) 394-6272

E-mail: kester@coe.ecu.edu

\section{LAYOUT EDITOR}

Michael Reece

Digital Technologies Specialist

Joyner Library

East Carolina University

Greenville, NC 57858

Tel: (252) 328-2897

Fax: (252) 328-2415

E-mail: reecem@ecu.edu

\section{ASSOCIATE ED. FOR WEB PUBLISHING}

Terry W. Brandsma

Information Technology Librarian

Jackson Library

UNC-Greensboro

P.O. Box 26170

Greensboro, NC 27402-6170

Tel: (336) 256-1218

Fax: (336) 334-5399

E-mail: twbrands@uncg.edu

\section{EDITOR EMERITUS}

Plummer Alston "Al" Jones, Jr.

LSIT, College of Education

East Carolina University

1108 Joyner Library

Greenville, NC 27858

Tel: (252) 328-6803

Fax: (252) 328-4368

E-mail: jonesp@mail.ecu.edu 